Hydrology and Earth System Sciences, 6(2), 153-166 (2002) C EGS

\title{
Influence of vegetation on SMOS mission retrievals
}

\author{
Khil-ha Lee, Eleanor J. Burke, W. James Shuttleworth and R. Chawn Harlow \\ Department of Hydrology and Water Resources, University of Arizona, Tucson, AZ 85721, USA
}

Email for corresponding author: shuttle@hwr.arizona.edu

\begin{abstract}
Using the proposed Soil Moisture and Ocean Salinity (SMOS) mission as a case study, this paper investigates how the presence and nature of vegetation influence the values of geophysical variables retrieved from multi-angle microwave radiometer observations. Synthetic microwave brightness temperatures were generated using a model for the coherent propagation of electromagnetic radiation through a stratified medium applied to account simultaneously for the emission from both the soil and any vegetation canopy present. The synthetic data were calculated at the look-angles proposed for the SMOS mission for three different soil-moisture states (wet, medium wet and dry) and four different vegetation covers (nominally grass, crop, shrub and forest). A retrieval mimicking that proposed for SMOS was then used to retrieve soil moisture, vegetation water content and effective temperature for each set of synthetic observations. For the case of a bare soil with a uniform profile, the simpler Fresnel model proposed for use with SMOS gave identical estimates of brightness temperatures to the coherent model. However, to retrieve accurate geophysical parameters in the presence of vegetation, the opacity coefficient (one of two parameters used to describe the effect of vegetation on emission from the soil surface) used within the SMOS retrieval algorithm needed to be a function of lookangle, soil-moisture status, and vegetation cover. The effect of errors in the initial specification of the vegetation parameters within the coherent model was explored by imposing random errors in the values of these parameters before generating synthetic data and evaluating the errors in the geophysical parameters retrieved. Random errors of $10 \%$ result in systematic errors (up to $0.5^{\circ} \mathrm{K}, 3 \%$, and $\sim 0.2 \mathrm{~kg} \mathrm{~m}^{-2}$ for temperature, soil moisture, and vegetation content, respectively) and random errors (up to $\sim 2{ }^{\circ} \mathrm{K}, \sim 8 \%$, and $\sim 2 \mathrm{~kg} \mathrm{~m}^{-2}$ for temperature, soil moisture and vegetation content, respectively) that depend on vegetation cover and soil-moisture status.
\end{abstract}

Keywords: passive microwave, soil moisture, vegetation, SMOS, retrieval

\section{Introduction}

Evaporation from bare soil or from soil beneath vegetation, infiltration into the soil and, thus, surface runoff, are important aspects of the land-surface water and energy balance that are controlled by near-surface soil moisture (often defined to be that present in the uppermost $5 \mathrm{~cm}$ ). Moreover, the uptake of soil water by vegetation for transpiration is, in part, controlled by soil moisture in the plants' rooting zone. Consequently, water and energy fluxes at the land-surface/atmosphere interface depend strongly on soil-moisture status (Kerr et al., 2001). Weather and climate are, in turn, influenced significantly by the local and regional availability of soil moisture via the evaporation and transpiration processes.

Many observational and modelling studies have shown the potential of passive microwave remote sensing, particularly L-band (1.4 GHz frequency), for estimating near-surface soil moisture (Calvet et al., 1995; Chanzy et al., 1997; Jackson et al., 1999; Teng et al., 1993; Wang et al., 1990; Wigneron et al., 2000). The development of robust retrieval algorithms for use with microwave brightness temperature measurements (e.g. Kerr et al., 2001; Njoku and Li, 1999; Wigneron et al., 2000) is essential if the retrieved soil moisture is to be used in, for example, climate analysis and prediction. Of particular uncertainty is the detailed effect of vegetation on the microwave emission from the soil surface. This is usually described by a single empirical parameter (Jackson and Schmugge, 1991), the optical depth of the vegetation (i.e. the opacity coefficient multiplied by the vegetation water content).

The European Space Agency (ESA) has selected the Soil Moisture Ocean Salinity (SMOS) mission (http:// www.cesbio.upstlse.fr/indexsmos.html), with a proposed launch date in the 2003-2005 time frame. The SMOS 
mission will be based on a dual polarisation, L-band radiometer with an innovative aperture synthesis concept (a two-dimensional interferometer) that can achieve an onthe-ground resolution of $\sim 50 \mathrm{~km}$ near the edge of the swath, coupled with multi-angular acquisition designed to deliver several key land-surface variables, namely, near-surface soil moisture, vegetation water content and effective temperature (Kerr et al., 2001; Wigneron et al., 2000). Wigneron et al. (2000) used simulated SMOS observations created by adding random and systematic errors to the same model proposed for the SMOS retrieval algorithm to test the accuracy of that algorithm. This model assumes that the optical depth of the vegetation does not depend on either the polarisation of the radiation or the look-angle of the sensor. It also assumes that the opacity coefficient is independent of temperature and vegetation water content.

Recent work has, however, shown that the opacity coefficient depends on both the gravimetric water content of vegetation (Wigneron et al., 1996, 2000; Le Vine and Karam, 1996) and the temperature (Wigneron et al., 2000). In addition, the canopy type and structure (Jackson and Schmugge, 1991), the polarisation (van de Griend and Owe, 1996) and wavelength of the radiation (Jackson and Schmugge, 1991) and the look-angle of the sensor (van de Griend and Owe, 1996) may also influence the opacity coefficient. Because the influence of these factors on the accuracy of the retrieval algorithm is currently uncertain, an accurate description of the role of vegetation is a significant need if near-surface soil moisture is to be estimated reliably. Moreover, in the context of the SMOS mission, understanding the role of vegetation on overall microwave emission is particularly important because the water content of the vegetation is one of the geophysical variables to be retrieved and the large $(\sim 50 \mathrm{~km})$ footprint of the sensor means that vegetation cover will rarely be homogeneous across the area sampled.

Lee et al. (2002) extended an existing coherent radiative transfer model (Wilheit, 1978) to create what is, in effect, a new approach to modelling the effect of vegetation on passive microwave emission and explored the ability of their model to describe field data. This approach results in a plausibly realistic model of intermediate complexity that captures, adequately, most of the important features of the microwave emission of vegetation, such as the influence of canopy type/structure, polarisation and wavelength of the radiation, look-angle of the sensor and temperature and water content of vegetation. In the present study, the modelling approach of Lee et al. (2002) was adopted and used to simulate synthetic, multi-angle, L-band microwave brightness temperatures of different soil-vegetationatmosphere systems to investigate (a) the retrieval capability of the proposed SMOS retrieval algorithm; and (b) the effect of heterogeneity in vegetation parameters on retrievals made with the proposed SMOS retrieval algorithm.

\section{Models and methods}

As outlined above, the strategy adopted in this study was to use the extended Wilheit (1978) model for a linked soil and vegetation system to generate synthetic microwave observations equivalent to those that (it is proposed) the SMOS mission will make for a range of soil wetness states and vegetation covers. These synthetic data were then regarded as the "true" observations and the accuracy of retrievals made using a retrieval algorithm similar to that proposed for SMOS was assessed. This retrieval algorithm optimises a simple representation of the effect of vegetation on microwave emission against the synthetic observations of surface brightness temperatures at each position in the footprint of the sensor.

\section{MULTI-LAYER MICROWAVE EMISSION MODEL}

The Wilheit (1978) model for the coherent propagation of electromagnetic radiation through a stratified medium relates the microwave brightness temperatures transmitted through the surface-air interface of the medium to the dielectric properties and temperature of the underlying layers. Lee et al. (2002) extended the application of the original Wilheit (1978) microwave emission model upwards through the vegetation canopy to create a model of the microwave emission that represents both the soil and the vegetation together. Thus, in this extended Wilheit (1978) model, the vegetation canopy is assumed to be infinite in horizontal extent and to be made up of homogeneous, planar layers of dielectric medium, which lie above similar homogeneous, planar layers of dielectric medium representing the soil.

In the Lee et al. (2002) model, the dielectric of the vegetation matter itself $\left(\varepsilon_{\mathrm{v}}\right)$ is calculated assuming a linear version of the Dobson et al. (1985) mixing model (hitherto used for soils), i.e. from:

$$
\varepsilon_{v}=\varepsilon_{d r y} V_{d r y}+\varepsilon_{f w} V_{f w}+\varepsilon_{b w} V_{b w}
$$

where $\varepsilon_{d r y}, \varepsilon_{f w}$, and $\varepsilon_{b w}$ are the dielectric permittivities, and $V_{d r y}, V_{f w}$, and $V_{b w}$ are the volume fractions of dry matter, free water and bounded water, respectively. It is assumed that $\varepsilon_{d r y}, \varepsilon_{f w}$, and $\varepsilon_{b w}$ are independent of the vegetation water content because they are the permittivities of a pure sample of the dry matter, free water and bounded water if such an ideal, pure sample were physically available. The dielectric permittivity of the mixed vegetation matter and air that make 
up the vegetation canopy $\left(\varepsilon_{c a n}\right)$ is assumed to be given by:

$$
\varepsilon_{\text {can }}^{\alpha}=\varepsilon_{v}^{\alpha} V_{V}+\varepsilon_{\text {air }}^{\alpha}\left(1-V_{v}\right)
$$

where $\varepsilon_{\text {air }}$ is the dielectric permittivity of air, $V_{v}$ is the fractional volume of vegetation elements per unit volume canopy and $\alpha$ is a "shape factor" (Note: In the case of the Dobson et al. (1985) mixing model for soils, $\alpha=0.65$.)

Equations (1) and (2) together calculate the dielectric constant for the canopy as a whole, and this amount of dielectric is then distributed vertically among the plane parallel layers above the soil. The heights of the top and bottom of the canopy, $h_{t}$ and $h_{b}$, respectively, are specified but gradual changes in dielectric permittivity are simulated around these levels by introducing broadening that follows a Gaussian distribution with standard deviations $\sigma_{t}$ and $\sigma_{b}$, respectively. This broadening reflects the natural variability between the individual plants that make up the canopy but its presence is also critical to the reliable operation of this coherent emission model. Avoiding sharp transitions in dielectric at canopy boundaries suppresses internal reflections within the canopy and the associated interference patterns in microwave emission. Lee et al. (2002) give a more detailed description of this phenomenon.

For the purpose of the present study and in the absence of any better information, the canopy dielectric permittivities $\varepsilon_{d r y}, \varepsilon_{f w}$ and $\varepsilon_{b w}$ in Eqn. (1) were set arbitrarily to the plausibly realistic values of $(2.0,0.1 j),(77.2,4.9 j)$ and $(4.0,1.0 j)$, respectively, while $V_{d r y}, V_{f w}$ and $V_{b w}$ were selected to represent a canopy which was $38 \%$ dry matter, the remainder being water assumed bound and free in equal amounts $\left(V_{d r y}=0.38\right.$; $\left.V_{f w}=V_{b w}=0.31\right)$. The volume fraction of vegetation in the canopy, $V_{v}$, and the shape factor, $\alpha$, are required to calculate $\varepsilon_{c a n}$ from Eqn. (2). In this study, the vegetation volume fraction was estimated from the fresh weight of the vegetation per unit area of canopy, $W_{\text {fresh }}$, and vegetation canopy structure parameters using the equation:

$$
V_{v}=f_{r}\left(\frac{W_{\text {fresh }}}{\left(h_{t}-h_{b}\right) \rho_{c}}\right)
$$

where $\rho_{c}$ is the density of the (fresh) vegetation in the canopy, estimated as $\left[\rho_{d r y} V_{d r y}+1000\left(1-V_{d r y}\right)\right] \mathrm{kg} \mathrm{m}^{-3}$, with $\rho_{d r y}=$ $330 \mathrm{~kg} \mathrm{~m}^{-3}$ (Ulaby and El-Rayes, 1987) the density of dry vegetation. (Note: in Eqn. (3), the re-normalisation factor, $f_{r}$ (usually close to unity), is included as a correction to allow for the possibility that (Gaussian) smoothing of the top and/ or bottom of the profile (unrealistically) distributes some of the canopy dielectric below ground level.) It is difficult to define the shape factor, $\alpha$, used in Eqn. (2) on the basis of existing field data. However, using parameter optimisation techniques and the same values for $\varepsilon_{d r y}, \varepsilon_{f w}$, and $\varepsilon_{b w}$ as in this study, Lee et al. (2002) obtained values of around 1.24 for a soybean canopy and, for the purpose or this exploratory study and in the absence of better information, this value was adopted for both horizontal and vertically polarised microwave radiation.

\section{SIMULATION OF SYNTHETIC SMOS DATA}

Synthetic microwave observations that correspond to those that will be provided during the SMOS mission were calculated using the extended Wilheit (1978) model (Lee et al., 2002) for a range of canopies and soil-moisture conditions. The calculations showed only limited sensitivity to the assumed nature of the soil and a soil particle size distribution of $75 \%$ sand and $5 \%$ clay was selected arbitrarily.

For the purpose of this modeling study, the temperature profiles in the soil and canopy were assumed uniform and set to $300 \mathrm{~K}$, and the soil moisture profile was also assumed to be uniform, with calculations made for soil moisture contents $\left(\theta_{s}\right)$ of $30 \%, 18 \%$, and $8 \%$, these being respectively referred to as "wet", "medium wet" and "dry". Calculations were made for four different types of vegetation broadly corresponding to "grass", "crop", "shrub" and "tree": the values of $h_{t}$ and the vegetation water content, $W_{v c}$, for these crops are given in Table 1. A simple canopy structure was assumed with, in each case, $\sigma_{t}$ set to $35 \%$ of $h_{t}$ and $\sigma_{b}$ and $h_{b}$ set to zero. The brightness temperature of the complete soilvegetation profile for both horizontal and vertical polarised L-band microwave radiation was then calculated using the extended Wilheit (1978) model (Lee et al., 2002) for swath angle dependent ranges of look-angles (Table 2) corresponding to those proposed for the SMOS mission.

\section{PROPOSED SMOS RETRIEVAL ALGORITHM}

The retrieval algorithm used in this study mimics that proposed for the SMOS mission and delivers three variables over land surfaces, namely, near-surface soil moisture, the optical depth of the vegetation cover (or, by implication, vegetation water content) and the effective temperature of the land surface (assumed to be the same for the vegetation and the soil surface). The retrieval is based on the Fresnel model for microwave emission and assumes two distinct sources, a single, thin layer of vegetation and the surface of the soil beneath.

Thus, in the retrieval algorithm, the microwave emissivity of the soil surface, $\mathrm{e}_{s}$ (which is equal to $\left(1-r_{s}\right.$ ), where $r_{s}$ is the reflectivity of the soil surface) relates the microwave brightness temperature of the soil, $T_{B}(\varphi p)$ (in deg K), to the 
Table 1. Surface conditions used when calculating the synthetic SMOS data. The soil effective temperature and the vegetation temperature were set to $300 \mathrm{deg} \mathrm{K}$.

\begin{tabular}{|c|c|c|c|c|c|c|}
\hline & $\begin{array}{l}\text { Soil } \\
\text { Wetness }\end{array}$ & $\begin{array}{l}\theta_{s} \\
(\% \text { vol. })\end{array}$ & $\begin{array}{l}\text { Temp. } \\
(\operatorname{deg} K)\end{array}$ & $\begin{array}{l}\text { Height } \\
\text { (m) }\end{array}$ & $\begin{array}{l}W_{v c} \\
\left(\mathrm{~kg} / \mathrm{m}^{2}\right)\end{array}$ & $\begin{array}{l}\text { Optical Depth } \\
\text { at Nadir }(\tau)\end{array}$ \\
\hline \multirow[t]{3}{*}{ Grass } & Wet & 30 & \multirow[t]{3}{*}{300} & \multirow[t]{3}{*}{0.3} & \multirow[t]{3}{*}{1.25} & 0.228 \\
\hline & Mid & 18 & & & & 0.251 \\
\hline & Dry & 8 & & & & 0.303 \\
\hline \multirow[t]{3}{*}{ Crop } & Wet & 30 & \multirow[t]{3}{*}{300} & \multirow[t]{3}{*}{1.0} & \multirow[t]{3}{*}{2.68} & 0.414 \\
\hline & Mid & 18 & & & & 0.431 \\
\hline & Dry & 8 & & & & 0.463 \\
\hline \multirow[t]{3}{*}{ Shrub } & Wet & 30 & \multirow[t]{3}{*}{300} & \multirow[t]{3}{*}{3.0} & \multirow[t]{3}{*}{4.17} & 0.627 \\
\hline & Mid & 18 & & & & 0.636 \\
\hline & Dry & 8 & & & & 0.652 \\
\hline \multirow[t]{3}{*}{ Tree } & Wet & 30 & \multirow[t]{3}{*}{300} & \multirow[t]{3}{*}{5.0} & \multirow[t]{3}{*}{7.50} & 1.121 \\
\hline & Mid & 18 & & & & 1.127 \\
\hline & Dry & 8 & & & & 1.138 \\
\hline
\end{tabular}

Table 2. The available look-angles as a function of the half-swath angle proposed for the SMOS mission. The synthetic observations were simulated using these look-angles at each half-swath angle (Wigneron et al., 1999)

\begin{tabular}{|c|c|}
\hline $\begin{array}{l}\text { Swath angle } \\
\text { (degrees) }\end{array}$ & $\begin{array}{l}\text { Look-angle } \\
\text { (degrees) }\end{array}$ \\
\hline 0.0 & 51.749 .146 .444 .341 .238 .737 .034 .231 .429 .427 .324 .121 .919 .617 .314 .912 .55 .12 .50 .0 \\
\hline 3.4 & 51.749 .246 .544 .341 .338 .837 .134 .431 .529 .627 .524 .422 .220 .017 .715 .413 .16 .34 .63 .8 \\
\hline 9.0 & 51.448 .946 .243 .441 .038 .636 .134 .331 .629 .726 .824 .822 .820 .817 .815 .914 .110 .410 .1 \\
\hline 11.2 & 49.847 .244 .442 .239 .937 .434 .933 .130 .428 .525 .723 .822 .020 .117 .515 .914 .512 .5 \\
\hline 12.3 & 48.746 .743 .941 .639 .336 .934 .331 .729 .928 .125 .323 .521 .720 .017 .616 .215 .014 .0 \\
\hline 14.4 & 47.745 .742 .940 .638 .335 .933 .431 .729 .127 .424 .823 .121 .620 .118 .217 .216 .5 \\
\hline 15.5 & $47.345 .242 .440 .237 .835 .433 .831 .328 .827,125.523 .121 .720 .419 .218 .017 .5$ \\
\hline 16.6 & 47.544 .842 .039 .737 .435 .933 .531 .029 .427 .025 .424 .022 .020 .819 .818 .818 .5 \\
\hline 18.6 & 46.744 .041 .939 .737 .535 .232 .931 .429 .127 .025 .624 .422 .821 .921 .120 .8 \\
\hline 19.6 & 46.343 .641 .639 .437 .235 .032 .831 .329 .127 .825 .924 .823 .422 .722 .021 .9 \\
\hline 20.6 & 45.944 .041 .239 .137 .034 .832 .731 .329 .228 .026 .225 .224 .023 .523 .1 \\
\hline 22.6 & 46.043 .441 .439 .437 .335 .333 .331 .430 .228 .627 .626 .425 .625 .325 .2 \\
\hline 23.6 & 5.743 .841 .239 .237 .235 .333 .431 .630 .529 .027 .827 .126 .526 .3 \\
\hline 25.4 & 45.843 .441 .539 .637 .836 .034 .232 .631 .230 .029 .128 .728 .4 \\
\hline 26.4 & 45.643 .241 .439 .637 .836 .134 .533 .031 .730 .629 .929 .529 .4 \\
\hline 28.1 & 46.544 .241 .940 .238 .637 .035 .133 .832 .832 .031 .531 .4 \\
\hline 29.9 & $46.244 ., 642.540 .438 .937 .536 .334 .934 .133 .633 .3$ \\
\hline 31.6 & 46.744 .643 .141 .239 .438 .237 .236 .135 .535 .2 \\
\hline 33.2 & 47.645 .743 .842 .140 .537 .0 \\
\hline
\end{tabular}


effective physical temperature of the soil, $T_{s}$ (in deg K) thus:

$$
T_{B}(\varphi, p)=e_{s}(\varphi, p) T_{s}
$$

where $e_{s}(\varphi, p)\left(1 \geq e_{s} \geq 0\right)$ is the polarised specular emissivity of the soil medium at angle $\varphi$ for polarisation state $p$ and is related to the soil dielectric constant via the Fresnel equations. The soil dielectric constant is calculated from the soil-moisture content and soil particle size distribution using Dobson's (1985) model. The simple model of Jackson and Schmugge (1991) is used to describe the effect of vegetation overlying the soil surface in terms of an equivalent optical depth, $\tau$, with:

$$
\tau=b W_{v c}
$$

where $b$ is the opacity coefficient, and $W_{v c}$ is the vegetation water content in $\mathrm{kg} \mathrm{m}^{-2}$.

By combining these simple concepts, Basharinov and Shutko (1975) presented a model for the microwave brightness temperature of an interface comprising a semiinfinite soil layer with physical temperature, $T_{s}$ (in ${ }^{\circ} \mathrm{K}$ ) and air-soil reflectivity $r_{s}(\varphi, p)$ with overlying vegetation with a physical temperature $T_{v}$ (in ${ }^{\circ} \mathrm{K}$ ), . This model is the basis for the algorithm, and the microwave brightness temperature is estimated as:

$$
\begin{aligned}
T_{B}(\varphi, p)= & {\left[1+r_{s}(\varphi, p) \Gamma(\varphi)\right][1-} \\
& \Gamma(\varphi)](1-\alpha) T_{v}+\left[1-r_{s}(\varphi, p)\right] \Gamma(\varphi) T_{s}
\end{aligned}
$$

where $\alpha$ is the single-scattering albedo of the soil surface, and $\Gamma(\varphi)$ is the transmissivity of the vegetation layer. This simple model is based on the following assumptions (Ulaby et al., 1986):

(a) the single-scattering albedo is assumed to be independent of $\varphi$ and $p$ and small $(0.04 \leq \alpha \leq 0.12)$;

(b) the air-vegetation reflectivity is assumed to be zero; and

(c) $r_{s}$ is set to the air-soil reflectivity rather than the vegetation-soil reflectivity because the average index of refraction of the vegetation layer is only slightly larger than that of air.

For simplicity, in the proposed SMOS retrieval algorithm, the soil temperature and vegetation temperature are assumed to be identical. Typically, the vegetation temperature drops below the soil temperature at night but, soon after sunrise, these temperatures are more similar: this assumption will influence the proposed timing of SMOS mission observations.

The SMOS mission will provide observations of surface brightness temperatures at several angles for each point on the ground, the number and selection of angles depending on the location of the point within the sensor footprint (Table 2). Over land surfaces, the mission will provide three geophysical variables, near-surface soil moisture, vegetation optical depth (assumed related to vegetation water content via Eqn. (5)) and an effective land-surface temperature (assumed equal for soil and vegetation). An optimisation technique will be used to deliver these geophysical variables from the multi-angle measurements of microwave brightness temperatures. The present paper seeks insight on the capability to retrieve these variables and the dependence of this on the nature of the vegetation overlying the soil independent of any possible shortcoming in the particular optimisation technique that is ultimately adopted for the SMOS retrieval. For this reason, the best multi-parameter optimisation algorithm available, the Shuffled Complex Evolution algorithm (SCE-UA; Duan et al., 1993) has been used in this study.

The SCE-UA is a global optimisation strategy designed to be effective and efficient for a broad class of complex global optimisation problems. It combines the strengths of the simplex procedure of Nelder and Mead (1965) with the concepts of controlled random search (Price, 1987), competitive evolution (Holland, 1975) and complex shuffling (Duan et al., 1993). All of these help to ensure that the information contained in a data sample is efficiently and thoroughly exploited in the attempt to find a global optimum. Hence, the SCE-UA algorithm has good convergence properties over a broad range of problems and a high probability of succeeding in its objective of finding the global optimum (Duan et al., 1993). The algorithm requires specification of a range of allowed values for each optimised variable. In this study, the range of values used in SCE-UA was $0-50 \%$ for soil moisture content (implicitly assumed constant with depth in the retrieval algorithm), 273$320 \mathrm{~K}$ for effective temperature (assumed equal for vegetation and soil in the retrieval algorithm) and $0-10 \mathrm{~kg}$ $\mathrm{m}^{-2}$ for vegetation water content.

It is possible to match the estimates of microwave emission given by the simple two-source model used in the SMOS retrieval algorithm and estimates given by the (assumed to be more realistic) extended Wilheit (1978) model (Lee et al., 2002) by selecting an appropriate value of the optical depth, $\tau$, or (for a specified value of vegetation water content) the opacity coefficient, $b$. Details of the derivation of the equivalent value of $\tau$ or $b$ are given in the Appendix. In practice, it proved necessary to derive and use look-angle dependent effective values of $b$ to achieve accurate retrieval of the geophysical variables when using the simple twosource model. 


\section{Results}

\section{MODEL EQUIVALENCE}

The central goal of the present study is to use the presumably better representation of the effect of vegetation on microwave emission in the extended Wilheit (1978) model to evaluate the simpler representation of vegetation in the two-source model that is the basis of the proposed SMOS retrieval algorithm. It is first necessary to ensure that these two models give equivalent representation of the microwave emission from bare soil when no vegetation is present. Figure 1 shows a comparison of the microwave brightness temperature for bare soil and the soil reflectivity calculated by the two models for three different conditions of soil moisture (i.e. $30 \%, 18 \%$ and $8 \%$ ). The agreement is excellent: the calculated values given by the two models are indistinguishable.

Figure 2 shows a comparison between the microwave brightness temperature for the combined soil-vegetation system calculated by the extended Wilheit (1978) model and the two-source model used in the SMOS retrieval for the three different soil moisture levels and for four different vegetation covers representing grass, crops, shrub and forest, with the vegetation parameters given in Table 1 . In this comparison, the value of the opacity coefficient, $b$, was determined using the approach given in the Appendix to ensure equivalence at the nadir. In general, using this value at greater look-angles in the Fresnel model results in increased estimates of microwave brightness temperatures for all of the simulated canopies and for both polarisation states. These differences are larger at greater look-angles for canopies with lower vegetation water content (the difference is greatest for grass and least for forest) and for vertically polarised radiation.

Clearly, it is possible to use the approach described in the Appendix to ensure equivalence between the two different models by modifying the value of $b$ at angles other than the nadir. In this way, it is possible to derive look-angle

\section{Brightness Temp.}
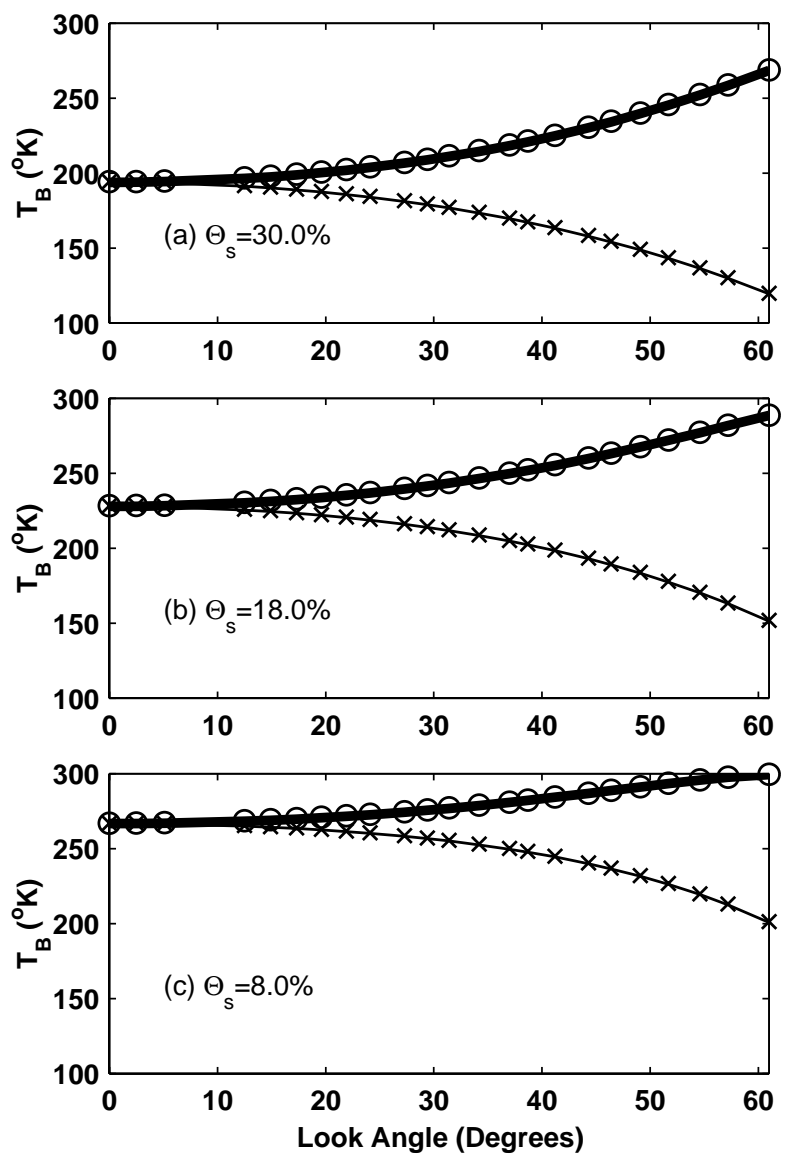

Reflectivity
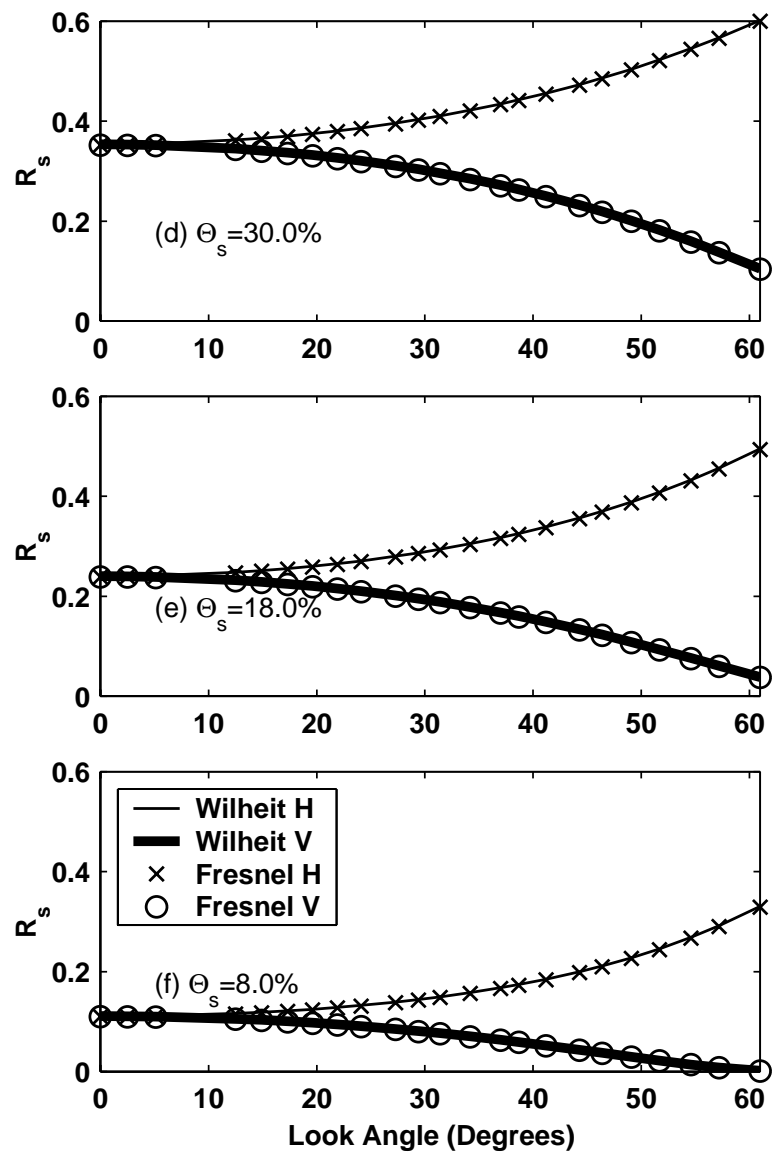

Fig. 1. Comparison of the microwave brightness temperature $\left({ }^{\circ} \mathrm{K}\right.$ ) and soil reflectivity (no units) calculated for bare soil using the extended Wilheit (1978) model and the two-source Fresnel model for three different soil wetness conditions. 

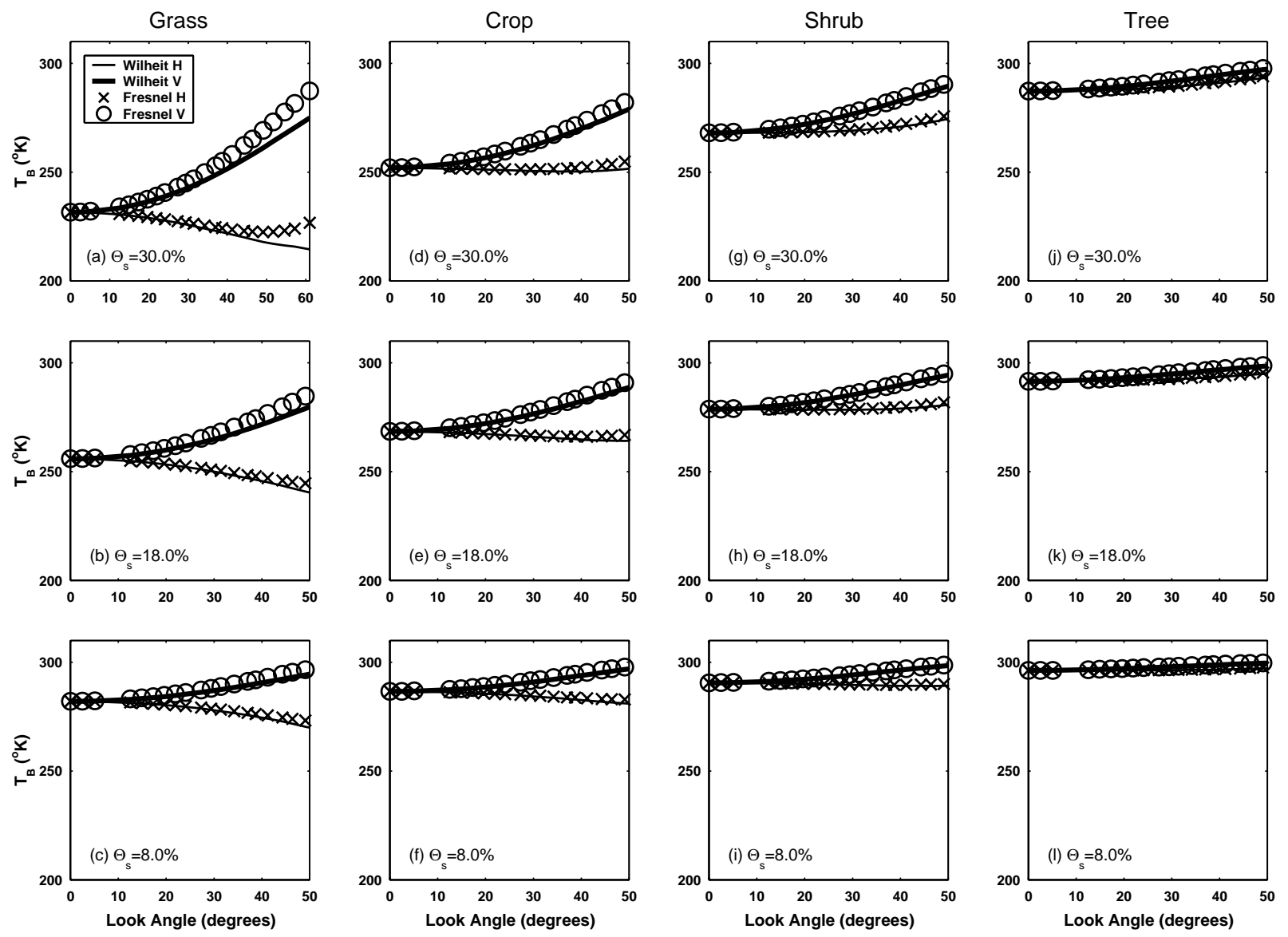

Fig. 2. Microwave brightness temperature $\left({ }^{\circ} \mathrm{K}\right)$ as a function of look-angle $\left({ }^{\circ}\right)$ for three different conditions of soil wetness $(8,18$, and $30 \%)$ and four different vegetation covers specified in Table 1 using a constant opacity coefficient, $b$, the value being specified to ensure equality between the two models at the nadir.

dependent functions specifying the value of $b$ needed to give accurate retrieval with the proposed SMOS retrieval algorithm for each of the selected soil-moisture levels and for each specified vegetation cover. Figure 3 shows the required equivalent value of $b$ needed for accurate retrieval as a function of look-angle and for both polarisation states. Consistent with Fig. 2, the variation in the required value of $b$ with look-angle is greater for vertically polarised radiation and for canopies with lower vegetation water content.

\section{RETRIEVAL ACCURACY FOR HOMOGENEOUS VEGETATION}

On the basis of these results, it is clear that to obtain truly accurate recovery using a retrieval algorithm based on the two-source Fresnel model in the presence of vegetation, it is necessary to allow for look-angle dependency in the effective value of $b$ that changes with the vegetation cover and soil-moisture status. The mechanics of making such an allowance is peripheral to the present study and, for the remainder of this paper, it is assumed that corrections for this systematic effect have been made and that the appropriate value of $b$ is being used in the retrieval. (In practice, perhaps these would be obtained from "look-up" tables applied in an iterative system). Outstanding issues are the accuracy of the retrieval of soil moisture, optical depth, and effective temperature from multi-angle data when using (in this study, state-of-the-art) optimisation techniques and the impact on that retrieval accuracy of having heterogeneous vegetation within a pixel.

The process of retrieval involves searching for that combination of soil moisture, optical depth and effective temperature which, when used in the Fresnel model, calculates microwave brightness temperatures (with appropriate, effective value of $b$ ) at the set of look-angles corresponding to a specified position in the footprint of SMOS that most nearly corresponds to the simulated observations calculated by the extended Wilheit (1978) model. In this study, searching for the preferred combination of variables involved randomly initiating the SCE-UA 
Grass
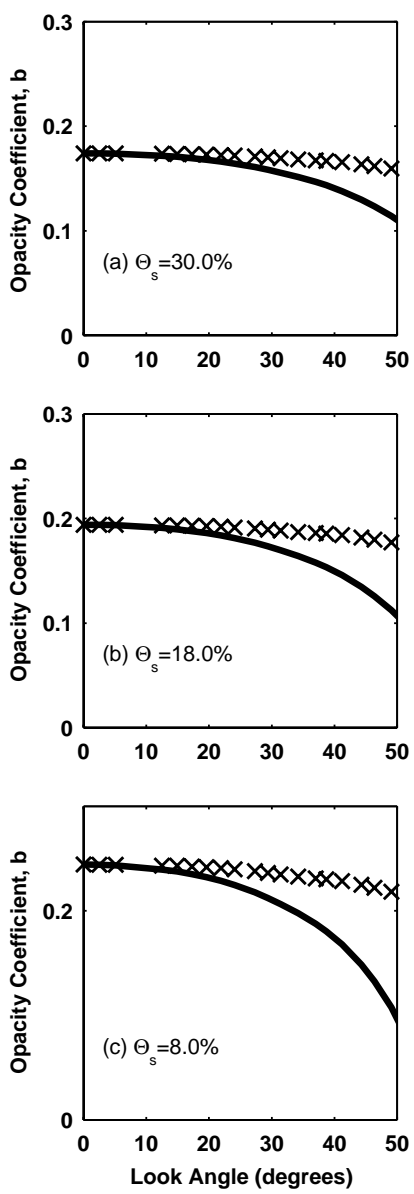

Crop
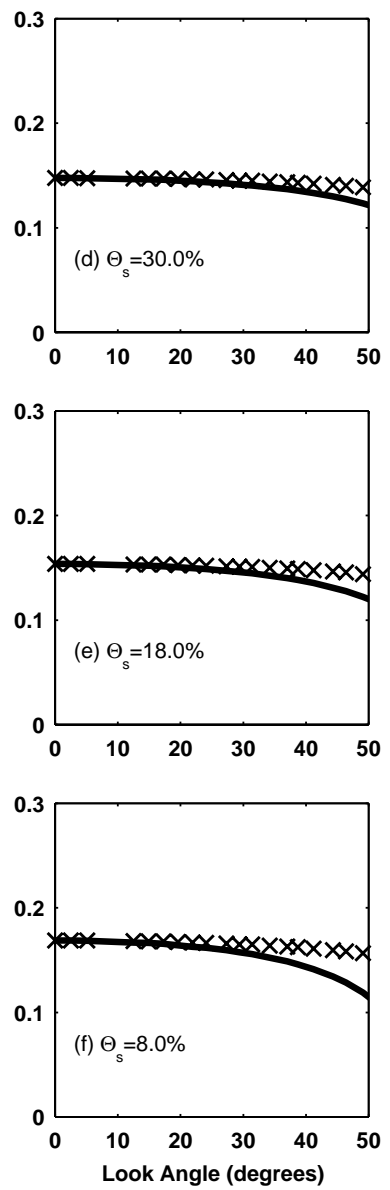

Shrub
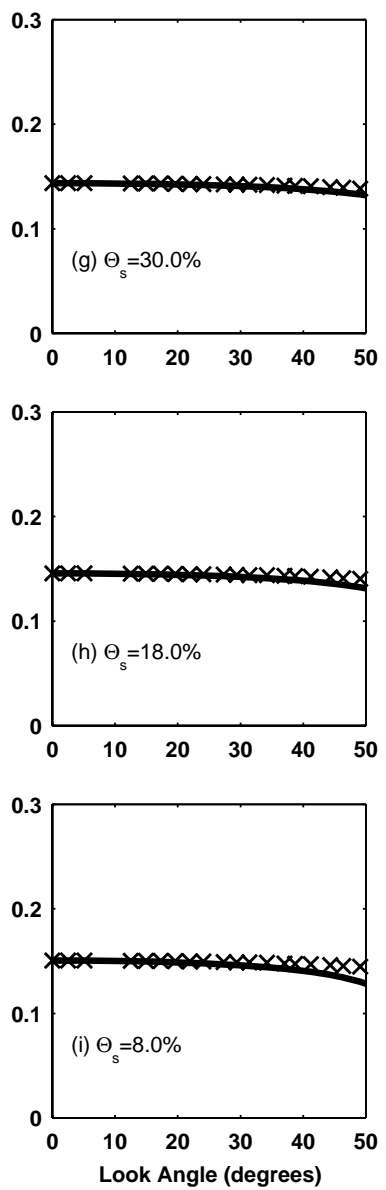

Tree
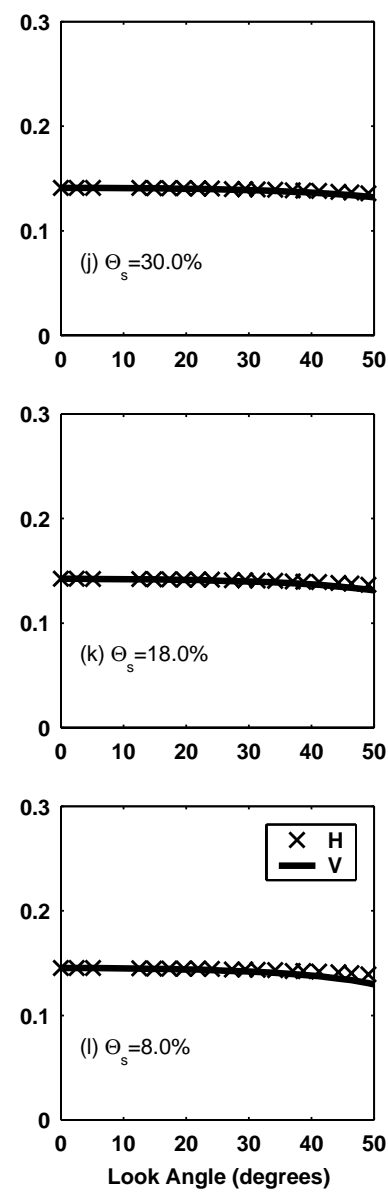

Fig. 3. The effective value of the opacity coefficient, $b$, required to make accurate retrievals with the two-source Fresnel model as a function of look-angle for three different soil wetness conditions and four different vegetation covers.

optimisation procedure within the allowed range of the required variables and allowing it to find that combination with minimum root mean square error (RMSE) between the brightness temperature calculated with the Fresnel model and the synthetic observations. In general, different random initiations of the SCE-UA algorithm resulted in the same set of values for the three required variables.

Figure 4 shows the results of applying the retrieval process with different vegetation covers and with different soil moisture states. In Fig. 4, the location of the sampled point within the SMOS footprint is specified on the horizontal axis as a function of the half-swath angle. Errors are given relative to the fixed equivalent values used in the forward calculations made with the extended Wilheit (1978) model, in percent, $10^{-1} \mathrm{~kg} \mathrm{~m}^{-2}$ and ${ }^{\circ} \mathrm{K}$ for soil moisture, vegetation water content and effective temperature, respectively. The retrieval results in very small errors in the retrieved values shown in Fig. 4; the errors are apparently haphazard and most obvious in the case of retrieved soil moisture. The presence of such "noise" confuses an interpretation of Fig. 4 but some general features are evident.

When changes in the effective value of $b$ are taken into account, the retrieval is very successful with errors in soil moisture typically less than $0.5 \%$, in vegetation water content less than $0.1 \mathrm{~kg} \mathrm{~m}^{-2}$ and with very small errors $(0.1 \mathrm{~K})$ in effective temperature. There is a tendency for the errors in the retrieved values, especially those in soil moisture, to be greater for bigger half-swath angles. This is, presumably, a consequence of the fact that the number of angles for which observations will be made in the SMOS mission reduces with half-swath angle. There is little evidence that retrieval errors change significantly with soil moisture status. However, there is some suggestion that the retrieval errors, at least for surface soil moisture, are greater for forest vegetation than for the other vegetation classes. 

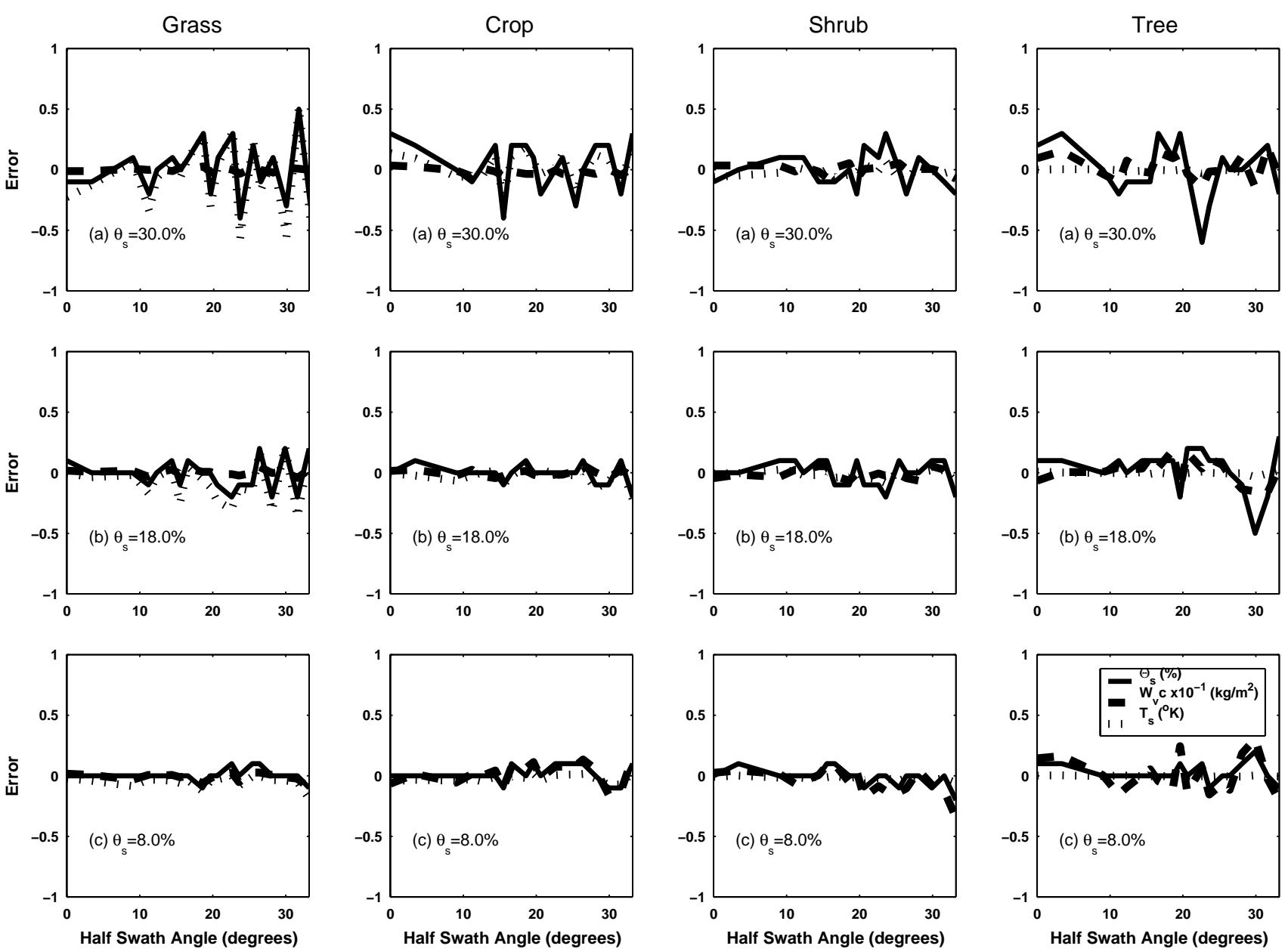

Fig. 4. Retrieved values of near-surface soil moisture, vegetation water content, and effective temperature obtained by optimising the twosource Fresnel model with the effective values of opacity coefficient given in Fig. 3, expressed as a function of half-swath angle for three different soil wetness conditions and four different vegetation covers.

\section{RETRIEVAL ACCURACY FOR HETEROGENEOUS}

\section{VEGETATION}

The accuracy of the retrievals depends on the nature of the overlying vegetation and requires appropriate specification of vegetation-related parameters. However, in practice, even within one land-cover type and particularly for pixel sizes $\sim 50 \mathrm{~km}$, there will be a large variation in the nature of the vegetation and hence the vegetation-related parameters. To explore the impact of realistic variability in the vegetation on the quality of retrieval, one thousand forward calculations of synthetic SMOS observations were made for selected half-swath angles, soil moisture states and vegetation covers, as before, with the vegetation parameters in the extended Wilheit (1978) model randomly selected around the values previously used following a Gaussian distribution with a standard deviation equal to $10 \%$ of their (previously fixed) value. Retrievals were then made in each case using the SCE-UA optimisation procedure and the mean and standard deviation of the retrieved geophysical variables calculated.

Figure 5 shows the mean of the retrieved variables obtained using the procedure just outlined. In this figure, the location of the sampled point within the SMOS footprint is again specified on the horizontal axis in terms of the halfswath angle, and errors are again given relative to the fixed equivalent values used in the forward calculations in percent for soil moisture, $10^{-1} \mathrm{~kg} \mathrm{~m}^{-2}$ for vegetation water content and ${ }^{\circ} \mathrm{K}$ for temperature, respectively. The most noticeable and significant result demonstrated by Fig. 5 is that random errors in the parameters used to specify the nature of the vegetation can result in systematic offsets in the retrieved values of soil moisture and vegetation water content but they have less impact on the retrieved value of effective temperature. The induced systematic errors are broadly similar for grass-, crop- and shrub-covered surfaces (less than $\sim 0.5 \%$ reduction in retrieved soil moisture and up to $\sim 0.2 \mathrm{~kg} \mathrm{~m}^{-2}$ increase in retrieved vegetation water content, 

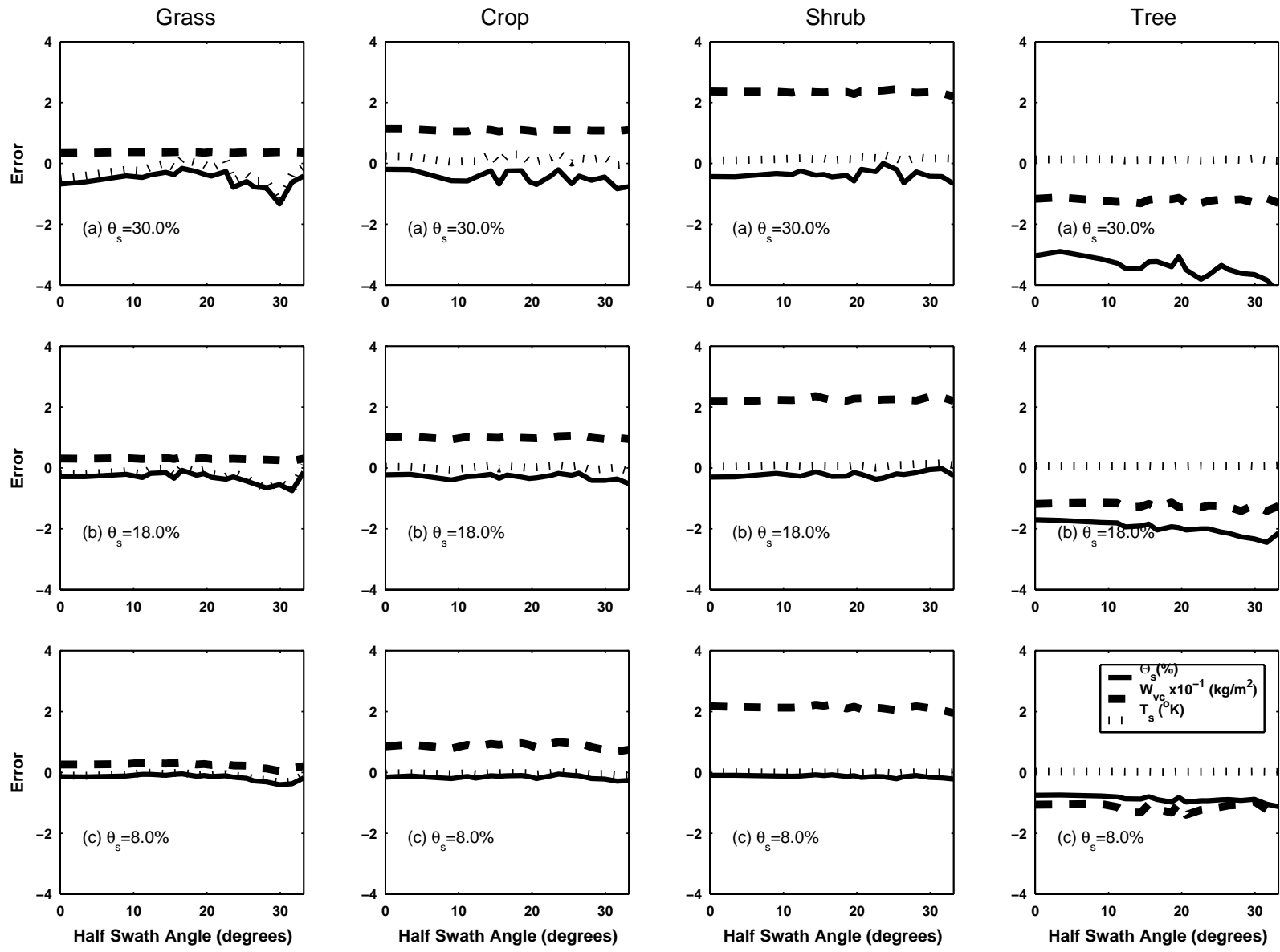

Fig. 5. Systematic error in retrieved values of near-surface soil moisture, vegetation water content, and effective temperature as a function of half-swath angle for three different soil wetness conditions and four different vegetation covers when $10 \%$ random errors are introduced into the specification of the vegetation-related parameters used when calculating the synthetic observations with the extended Wilheit (1978) model.

depending on the vegetation cover), but are noticeably different in the case of forest cover $(\sim 1-4 \%$ reduction in retrieved soil moisture, depending on the wetness of the soil, and a $\sim 0.15 \mathrm{~kg} \mathrm{~m}^{-2}$ decrease in vegetation water content). In the case of forest cover, there is also some tendency for the systematic error in retrieved soil moisture to increase with half-swath angle, presumably because the sampled range of look-angles changes and, on average, the absolute values increase as the half-swath angle increases.

Imposing random errors in vegetation-related parameters generates both systematic and random errors in the retrieved variables. Table 3 gives the systematic bias and standard deviation of the retrieved values of soil moisture, vegetation water content and effective temperature at zero swath angle when $10 \%$ random errors are introduced into the vegetationrelated parameters used to calculate the synthetic observations using the extended Wilheit model. In general, the absolute values of the systematic errors are small and less than $1 \%$ by volume in soil water content (but up to $3 \%$ for forest cover over moist soil), less than $0.1 \mathrm{~kg} \mathrm{~m}^{-2}$ in vegetation water content (but up to $0.1 \mathrm{~kg} \mathrm{~m}^{-2}$ for shrub cover over moist soil) and typically $\sim 0.1 \mathrm{~K}$ in temperature (but up to $\sim 0.5 \mathrm{~K}$ for grass cover over moist soil). The random errors in retrieved variables (here in all cases expressed in terms of the standard deviation of retrieved values) differ with the retrieved variable. The random error in soil moisture increases with soil wetness, being typically $\sim 1-3 \%$ for grass, crop and shrub cover but is much larger both in absolute terms (up to 8\%) and as a fraction of soilwater content for forest cover. Perhaps not surprisingly, the random error in vegetation water content generated by introducing $10 \%$ errors in vegetation parameters (largest in absolute terms for forest cover) is high in percentage terms (25--40\%) for all cover types and soil-moisture conditions. While the random error in retrieved temperature is small for forest cover, it increases to $\sim 2 \mathrm{~K}$ for grass cover over moist soil.

It is interesting to see how the systematic errors and the 
Table 3. Bias and standard deviation (SD) of retrieved surface variables at zero-swath angle when vegetationrelated parameters are randomly selected following a Gaussian distribution with standard deviation of 10\%.

\begin{tabular}{lcllllll}
\hline $\begin{array}{l}V_{v c} \\
\left(\mathrm{~kg} \mathrm{~m}^{-2}\right)\end{array}$ & $\begin{array}{c}\theta_{s} \\
(\%)\end{array}$ & $\begin{array}{l}\text { Bias in } \theta_{s} \\
(\%)\end{array}$ & $\begin{array}{l}\text { SD in } \theta_{s} \\
(\%)\end{array}$ & $\begin{array}{l}\text { Bias in } W_{v c} \\
\left(\mathrm{~kg} \mathrm{~m}^{-2}\right)\end{array}$ & $\begin{array}{l}S D \text { in } W_{v c} \\
\left(\mathrm{~kg} \mathrm{~m}^{-2}\right)\end{array}$ & $\begin{array}{l}\text { Bias in } T_{s} \\
\left({ }^{\circ} \mathrm{K}\right)\end{array}$ & $\begin{array}{l}\text { SD in } T_{s} \\
\left({ }^{\circ} \mathrm{K}\right)\end{array}$ \\
\hline Grass & 30 & -0.7 & 2.5 & 0.04 & 0.394 & -0.49 & 2.17 \\
1.25 & 18 & -0.3 & 1.3 & 0.03 & 0.380 & -0.20 & 1.08 \\
& 8 & -0.1 & 0.6 & 0.03 & 0.367 & -0.10 & 0.32 \\
Crop & 30 & -0.2 & 2.5 & 0.11 & 0.965 & 0.25 & 1.05 \\
2.68 & 18 & -0.2 & 1.3 & 0.10 & 0.936 & 0.04 & 0.49 \\
& 8 & -0.2 & 0.6 & 0.08 & 0.903 & -0.03 & 0.13 \\
Shrub & 30 & -0.4 & 2.2 & 0.23 & 1.700 & 0.10 & 0.38 \\
4.17 & 18 & -0.3 & 1.2 & 0.22 & 1.673 & 0.05 & 0.18 \\
& 8 & -0.1 & 0.5 & 0.21 & 1.647 & -0.00 & 0.05 \\
Tree & 30 & -3.0 & 8.3 & -0.11 & 2.092 & 0.13 & 0.25 \\
7.50 & 18 & -1.7 & 4.8 & -0.12 & 2.075 & 0.08 & 0.13 \\
& 8 & -0.8 & 2.2 & -0.10 & 2.059 & 0.02 & 0.04 \\
\hline
\end{tabular}

associated random errors in retrieved values change with the size of random errors introduced into the vegetationrelated parameters. Recognizing that the systematic errors for grass-, crop- and shrub-covered surfaces are similar and also broadly similar for different soil moisture states, Fig. 6 shows the error in the retrieved variables for crop and forest cover for medium soil moisture status $(18 \%)$ as a function of the standard deviation of the random error added to the vegetation parameters in the extended Wilheit (1978) model. The mean temperature is always retrieved very accurately,
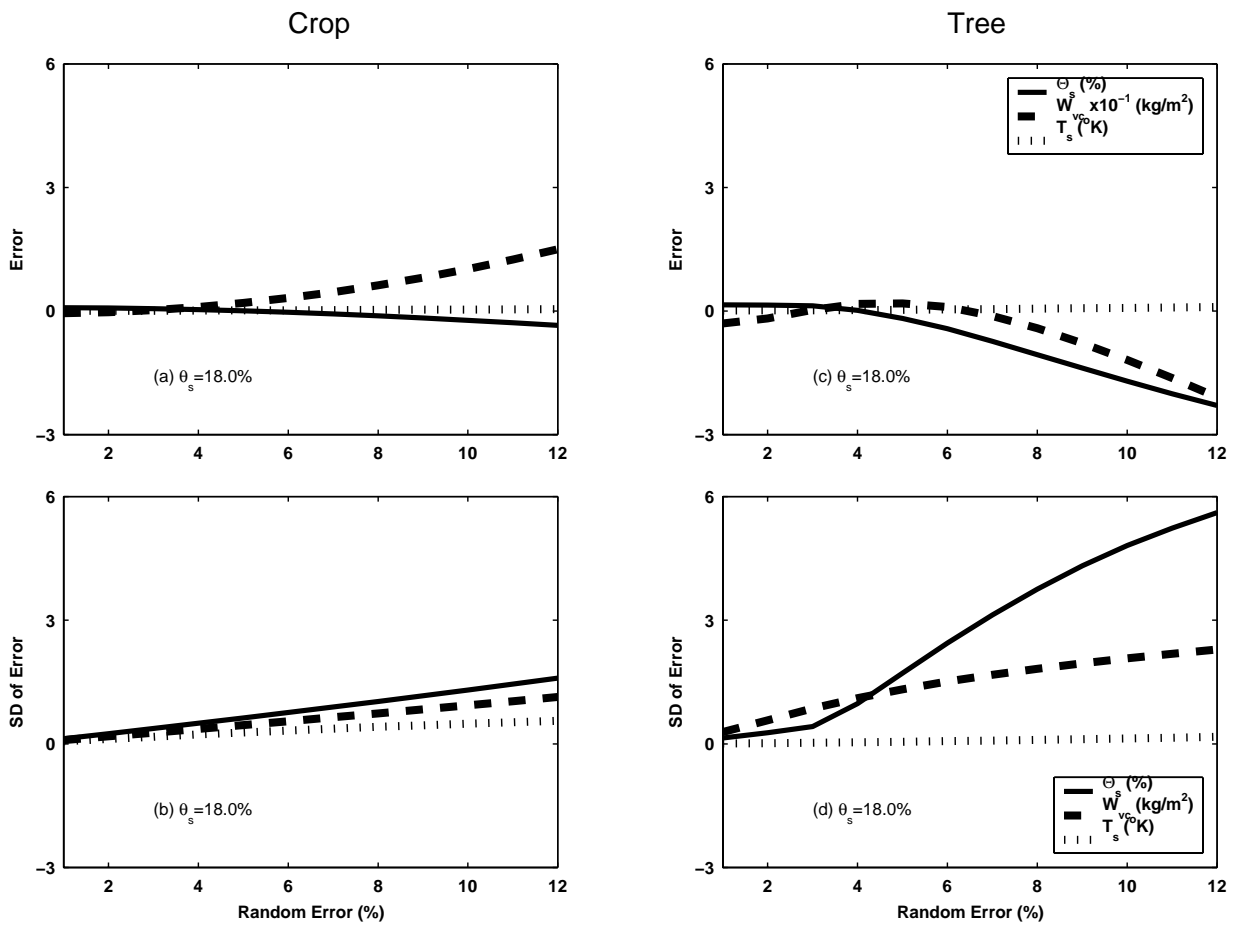

Fig. 6. Systematic error (given as a percentage for soil moisture and vegetation water content and absolute value for effective temperature) and random error (given as a percentage of the mean value) in retrieved values of near-surface soil moisture, vegetation water content, and effective temperature as a function of the random errors in the specification of vegetation-related parameters when calculating synthetic observations with extended Wilheit (1978) model. Results are given for crop cover and forest cover and for a soil-moisture status of 18\%. 
regardless of the imposed errors in vegetation parameters. However, as the standard deviation of the random errors increases, the systematic error in the retrieved soil moisture and vegetation water content increases. The systematic error in the retrieved soil moisture increases more significantly than in vegetation water content for forest cover, and viceversa for the crop cover. This might be expected because of the greater sensitivity of the microwave brightness temperature to the soil moisture when covered with vegetation with less water content. The random errors in soil moisture and vegetation water content also increase as the random error in vegetation parameter increases, and become large, particularly in the case of forest cover.

\section{Summary and conclusions}

This study investigated the potential sensitivity of the values of near-surface soil moisture, vegetation water content and effective temperature retrieved from multi-angle microwave radiometer observations to the presence and nature of vegetation cover, with the proposed SMOS mission used as a case study. The strategy adopted was, firstly, to simulate the range of observations of microwave brightness temperature that (it is proposed) SMOS will make using an extended version of the Wilheit (1978) model (Lee et al., 2002) for three different soil moisture states (wet, medium and dry) and four different vegetation covers (grass, crop, shrub and forest). Then, the required values were found using a retrieval approach that mimicked that proposed for the SMOS mission in that it assumed a two-source Fresnel representation of microwave emission and used advanced optimization techniques.

The primary conclusions of the present study are as follows:

- When describing the microwave emission of bare soil, the simple two-source (Fresnel) model of microwave emission used in the proposed SMOS retrieval and the extended Wilheit (1978) model are consistent.

- When describing the microwave emission of vegetationcovered soil, the simple two-source model of microwave emission is not consistent in detail with the extended Wilheit (1978) model and cannot therefore be used to retrieve accurate values of near-surface soil moisture, vegetation water content and effective temperature unless effective values of the opacity coefficient, $b$, that vary with look-angle, soil moisture status and vegetation cover are used.

- If effective values of the opacity coefficient are used, the proposed SMOS retrieval algorithm gives very good retrievals, with errors in soil moisture typically of the order of $1 \%$; in vegetation water content less than $0.5 \%$; and with very small errors in effective temperature.

- With multi-angle microwave radiometers and a retrieval algorithm based on the simple two-source (Fresnel) model, heterogeneity within the vegetation cover (as represented by introducing $10 \%$ random error in vegetation parameters) can result in both systematic and random errors in retrieved values which are vegetation cover and soil-moisture status dependent.

- Systematic errors in retrieved variables induced by $10 \%$ random errors in vegetation parameters are up to $0.5 \mathrm{~K}$ for temperature (worst for grass cover and moist soil), up to $3 \%$ in soil moisture (worst for forest cover and moist soil), and up to $\sim 0.2 \mathrm{~kg} \mathrm{~m}^{-2}$ for vegetation content (worst for shrub cover). Random errors so induced are up to $\sim 2^{\circ} \mathrm{K}$ for temperature (worst for grass cover and moist soil), up to $\sim 8 \%$ in soil moisture (worst for forest cover and moist soil), and up to $\sim 2 \mathrm{~kg} \mathrm{~m}^{-2}$ for vegetation content (worst for forest cover).

- The size of both systematic and random errors increases with the heterogeneity in vegetation cover (as represented by random error in vegetation parameters) for all cover types; the systematic error in the retrieved soil moisture increases more significantly than in vegetation water content for forest cover, and vice-versa for the crop cover.

\section{Acknowledgements}

Primary support for Khil-ha Lee and Dr. Eleanor J. Burke while preparing this paper was provided from NOAA project NA96GP0412. Additional support for Dr. W. James Shuttleworth was provided from NASA project NAG81531. The editorial assistance provided by Corrie Thies is appreciated.

\section{References}

Basharinov, A.E. and Shutko, A., 1975. Simulation studies of the SHF radiation of soils under moist conditions. NASA Tech. Translation TT F-16.

Calvet, J.-C., Wigneron, J.-P., Chanzy, A. and Haboudane, D., 1995. Retrieval of surface parameters from microwave radiometry over open canopies at high frequencies. Remote Sens. Environ., 53, 46-60.

Chanzy, A., Schmugge, T.J., Calvet, J.-C., Kerr, Y., van Oevelen, P., Grosjean, O. and Wang, J.R., 1997. Airborne microwave radiometry on a semi-arid area during Hapex-Sahel. J. Hydrol., 188-189, 285-309.

Dobson, M.C., Ulaby, F.T., Hallikainen, M.T. and El-Rayes, M.A., 1985. Microwave behavior of wet soil, 2: dielectric mixing models. IEEE Trans. Geosci. Remote Sens., 23, 35-46.

Duan, Q.Y., Gupta, V.K. and Sorooshian, S., 1993. Shuffled Complex Evolution approach for effective and efficient global minimization. J. Optimiz.Theor. Appl., 76, 501-521. 
Holland, J.H., 1975. Adaptation in Natural and Artificial Systems. University of Michigan Press, Ann Arbor, Michigan.

Jackson, T.J. and Schmugge, T.J., 1991. Vegetation effects on the microwave emission of soils. Remote Sens. Environ., 36, $203-$ 212.

Jackson, T.J., Le Vine, D.M., Hsu, A.Y., Oldak, A., Stark, P.J., Swift, C.T., Isham, J.D. and Haken, M., 1999. Soil moisture mapping at regional scales using microwave radiometry: the Southern Great Plains Hydrology Experiment. IEEE Trans Geosci. Remote Sens., 37, 2136-2151.

Kerr, Y.H., Waldteufel, P., Wigneron, J.-P., Martinuzzi, J.-M., Font, J. and Berger, M., 2001. Soil moisture retrieval from space: the Soil Moisture and Ocean Salinity (SMOS). IEEE Trans. Geosci. Remote Sens., 39, 1729-1735.

Kirdyashev, K.P., Chukhlantsev, A.A. and Shutko, A.M., 1979. Microwave Radiation of the earth's surface in the presence of a vegetation cover. Radiotekhnika I Elektronika, 24, 256-264 (Engl. Transl.)

Le Vine, D.M. and Karam, M.A., 1996. Dependence of attenuation in a vegetation canopy on frequency and plant water content. IEEE Trans. Geosci. Remote Sens., 34, 1090-1096.

Lee, K.H., Harlow, R.C., Burke, E.J. and Shuttleworth, W.J., 2002. Application of a plane-stratified emission model to predict the effects of vegetation in passive microwave radiometry. Hydrol. Earth Syst. Sci., 6, 139-151.

Nelder, J.A. and Mead, R.A., 1965. A simplex method for function minimization. Comput. J., 7, 308-313.

Njoku, E.G. and Li, L., 1999. Retrieval of land surface parameters using passive microwave measurements at 6-18 GHz. IEEE Trans. Geosci. Remote Sens., 37, 79-93.

Price, W.L., 1987. Global optimization algorithms for a CAD workstation. J. Optimiz. Theor. Appl., 55, 133-146.

Teng, W.L., Wang, J.R. and Doraiswamy, P.C., 1993. Relationship between satellite microwave radiometric data, antecedent precipitation index, and regional soil moisture. Int. J. Remote Sens., 14, 2483-2500.

Ulaby, F.T. and El-Rayes, M.A., 1987. Microwave dielectric spectrum of vegetation. 2, dual dispersion. IEEE Trans. Geosci. Remote Sens., 25, 550-557.

Ulaby, F.T., Moore, R.K. and Fung, A.K., 1986. Microwave Remote Sensing-Active and Passive. Addison-Wesley, Reading, MA.

van de Griend, A.A. and Owe, M., 1996. Measurement and behavior of dual-polarization vegetation optical depth and single scattering albedo at 1.4 and $5 \mathrm{GHz}$ microwave frequencies. IEEE Trans. Geosci. Remote Sens., 34, 957-965.

Wang, J.R., Shiue, J.C., Schmugge, T.J. and Engman, E.T., 1990. The L-band PBMR measurements of surface of soil moisture. IEEE Trans. Geosci. Remote Sens., 28, 906-913.

Wigneron, J.-P., Calvet, J.-C. and Kerr, Y.H., 1996. Monitoring water interception by crop fields from passive microwave observations. Agr. Forest Meteorol., 80, 177-194.

Wigneron, J.-P., Waldteufel, P., Chanzy, A., Calvet, J.-C., Marloie, O., Hanocq, J.-F. and Kerr, Y.H., 1999. Retrieval capabilities of L-band 2-D interferometric radiometry over land surfaces (SMOS mission). In: Microwave Radiometry and Remote Sensing of the Earth's Surface and Atmosphere, VSP, The Netherlands, $552 \mathrm{pp}$.

Wigneron, J.-P.,Waldteufel, P., Chanzy, A., Calvet, J.-C. and Kerr, Y., 2000. Two-dimensional microwave interferometer retrieval capabilities over land surfaces (SMOS mission). Remote Sens. Environ., 73, 270-282.

Wilheit, T.T., 1978. Radiative transfer in a plane stratified dielectric. IEEE Trans. Geosci. Electron., 16, 138-143.

\section{APPENDIX}

\section{DERIVATION OF EQUIVALENT OPTICAL DEPTH}

The extended Wilheit (1978) model (Lee et al., 2002) calculates the microwave brightness temperature, $T_{B, a l l}$, of the complete soil-vegetation-atmosphere interface as:

$$
T_{B, \text { all }}=\sum_{i=1}^{N} f_{i} \cdot T_{i}
$$

where $T_{i}$ is the temperature of the $i^{\text {th }}$ layer, $f_{i}$ is the fraction of energy absorbed from an incident microwave by the $i^{t h}$ layer of dielectric represented in the model and $N$ is the number of the layers in the semi-infinite medium. A number, $N_{s}$, of these layers of dielectric represent the underlying soil. Hence, the effective reflectivity, $r$, and emissivity, $e_{s}$, of the soil surface within the overall description of the Lee $e t$ al. (2002) model are given by:

$$
r_{s}=\left(1-e_{s}\right)=1-\sum_{i=1}^{N_{s}} f_{i}
$$

while the effective microwave brightness temperature of the soil surface is:

$$
T_{B, \text { soil }}=\sum_{i=1}^{N_{s}} f_{i} \cdot T_{i}
$$

In the simpler, two-layer Fresnel model of the soilvegetation-atmosphere interface, vegetation is treated as an absorbing layer with a transmissivity, $\Gamma$, which is described in terms of an optical depth, $\tau$, (Kirdyashev et al., 1979) that depends on the vegetation dielectric properties, the plant shape or structure, the wavelength and polarisation of the radiation, and the look angle, $\varphi$, (Jackson and Schmugge, 1991). Thus:

$$
\Gamma=\exp (-\tau \sec \varphi)
$$

In this expression, $\tau$ is often written (see, for instance, Jackson and Schmugge, 1991) as:

$$
\tau=b W_{v c}
$$

where $W_{v c}$ is the vegetation water content $\left(\mathrm{kg} \mathrm{m}^{-2}\right)$ and $b$, the opacity coefficient, is a parameter that represents all of the other influences of vegetation on optical depth.

Assuming there is no scattering of radiation at long wavelengths, the two-layer model gives the overall microwave brightness temperature of the soil-vegetationatmosphere interface as: 


$$
T_{B, a l l}=\left(1+r_{s} \Gamma\right)(1-\Gamma)(1-\alpha) T_{V}+\left(1-r_{s}\right) \Gamma T_{s}
$$

where $\alpha$ is the single scattering albedo, and $T_{v}$ is the temperature of the vegetation canopy. Equation (A6) can be rearranged to give:

$$
\Gamma^{2}+\frac{\left[\left(1-r_{s}\right)(1-\alpha) T_{v}-\left(1-r_{s}\right) T_{s}\right]}{(1-\alpha) r_{s} T_{v}} \Gamma-\frac{\left[T_{B, a l l}-(1-\alpha) T_{v}\right]}{(1-\alpha) r_{s} T_{v}}=0
$$

Hence, if the value of $\alpha$ is known or assumed, $\Gamma$ can be found for known values of temperatures and $r_{s}$ as the (real) solution of the quadratic Eqn. (A7). The effective value of the optical depth, $\tau$, and the opacity coeficient, $b$, in the two-level model can then be calculated from Eqns. (A4) and (A5), respectively. In this way, the dependence of $\tau$ and $b$ in the simpler two-source model can be investigated in terms of vegetation characteristics, as specified by parameters in the extended Wilheit (1978) model (Lee et al., 2002). 